\title{
S-(1,2-Dicarboxyethyl)glutathione and Glutathione in Lens and Liver of Naphthalene-Treated Rabbits
}

\author{
Megumi Takemura ${ }^{1}$, Hisayuki Ueno ${ }^{1}$ and Hiroyuki Kodama ${ }^{2}$ \\ 1 Department of Ophthalmology \\ 2 Department of Chemistry \\ Kochi Medical School, Kohasu, Oko-cho, Nankoku-shi, Kochi 783, Japan
}

Summary: The determination of S-(1,2-dicarboxyethyl)glutathione and reduced glutathione (GSH) in the rabbit lens and liver was developed using an isotachophoretic analyser.

The recovery of S-(1,2-dicarboxyethyl)GSH from the rabbit liver after ion-exchange treatment was $96.8 \pm 11.3 \%$ $(\mathrm{n}=3)$. The contents of S-(1,2-dicarboxyethyl)GSH in the rabbit lens and liver were $219.9 \pm 29.1(\mathrm{n}=5)$ and $44.0 \pm 13.5(\mathrm{n}=8) \mathrm{nmol} / \mathrm{g}$, respectively.

The contents of S-(1,2-dicarboxyethyl)GSH in the lens and GSH in the lens and liver of naphthalene-treated rabbits was also determined by this method 24 hours after naphthalene administration, at which time the axial opacity "spichen" was observed at the equatorial region of the lens. The content of S-(1,2-dicarboxyethyl)GSH in the lens decreased in proportion to the content of GSH.

During the further development of true lens opacity after naphthalene administration, the S-(1,2-dicarboxyethyl)GSH content further compared with that in the spichen stage, but the S-(1,2-dicarboxyethyl)GSH content of the lens that did not develop true opacity after naphthalene administration returned to the normal level. The change of S-(1,2-dicarboxyethyl)GSH content of the lens in the spichen and true opacity stages coincided with that of GSH content.

On the other hand, the content of GSH of the liver decreased markedly until 24 hours after naphthalene administration, then returned to normal, irrespective of whether true opacity did or did not subsequently develop.

\section{Introduction}

In 1963, S-(1,2-dicarboxyethyl)glutathione and S-(1,2dicarboxyethyl)- $L$-cysteine were isolated from calf lens. Their chemical structures were identified by comparison with the synthetic compounds, and it has been reported that S-(1,2-dicarboxyethyl)GSH was present at a higher concentration in the lens than in the other tissues of animals (1).

S-(1,2-dicarboxyethyl)- $L$-cysteine has also been identified in the urine and kidney of guinea pig (2), in ox brain and spinal cord (3) and in ox liver and kidney (4).

More recently, S-(1,2-dicarboxyethyl)GSH was determined by using high performance liquid chromatography (HPLC) after pre-column reaction with 2,4-dinitrofluorobenzene, and a decrease of S-(1,2-dicarboxyethyl)GSH in rat lens during galactose cataract formation has been reported (5).

However, the physiological significance and biochemical role of S-(1,2-dicarboxyethyl)GSH are still unknown.

Herein, we describe the determination of S-(1,2-dicarboxyethyl)GSH and reduced glutathione (GSH) using an isotachophoretic analyser (6-9), and we establish the correlation between their concentrations in the lens and cataract formation in rabbits during naphthalene treatment.

\section{Materials and Methods \\ Materials}

Reduced glutathione (GSH) and glutathione disulfide (GSSG) were obtained from Sigma. S-(1,2-dicarboxyethyl)-L-cysteine was synthesized as reported previously (5). S-(1,2-dicarboxyethyl)glutathione was kindly supplied from $\operatorname{Dr} K$. Ogata of the Senju Pharmaceutical Co. (Osaka). All other chemicals used were of analytical grade.

\section{Tissue preparation}

Male and female albino rabbits, weighing approximately $2 \mathrm{~kg}$ were used. Rabbits were killed by injecting air into an ear vein, and lens and liver were then removed, blotted, weighed, and used immediately.

\section{Assay of S-(1,2-dicarboxyethyl)glutathione}

Lens $(0.5 \mathrm{~g})$ and liver $(1.0 \mathrm{~g})$ were homogenized in $3 \mathrm{ml}$ of methanol- $\mathrm{H}_{2} \mathrm{O}\left(70: 30\right.$; by vol.) at $0^{\circ} \mathrm{C}$ for $10 \mathrm{~min}$, and centrifuged at $9000 \mathrm{~g}$ (lens) and $3000 \mathrm{~g}$ (liver) for $15 \mathrm{~min}$.

Each supernatant was adjusted to $\mathrm{pH} 5.0$ with $2 \mathrm{~mol} / \mathrm{l}$ acctic acid, transferred to a column containing $5 \mathrm{ml}$ of Amberlite IRA-68 (ace- 




Fig. 1 Isotachophoretic runs of authentic $10 \mathrm{nmol} \mathrm{S}$-(1,2-dicarboxyethyl)-L-cysteine, S-(1,2-dicarboxyethyl)GSH, GSSG and GSH. Analytical conditions: the leading electrolyte was $0.01 \mathrm{~mol} / 1$ hydrochloric acid and $\beta$-alanine $\mathrm{pH} 3.1$ (a) and $\mathrm{pH} 2.8$ (b). Terminal electrolyte was $0.01 \mathrm{~mol} / 1$ caproic acid.

For other conditions, see materials and methods.

tate form, $200-200$ mesh), washed with $30 \mathrm{ml}$ of $2 \mathrm{~mol} / \mathrm{l}$ acetic acid and eluted with $50 \mathrm{ml}$ of $1 \mathrm{~mol} / \mathrm{l}$ hydrochloric acid. The eluate was evaporated under reduced pressure. The residue was dissolved in $2 \mathrm{ml}$ of water, adjusted to $\mathrm{pH} 9.0$ with $2 \mathrm{~mol} / \mathrm{l}$ ammonia solution and transferred to a column containing $5 \mathrm{ml}$ of Diaion SA (formic form of sulphonated cation exchanger, mesh 100, Mitsubishi Kasei Co., Tokyo), washed with water and eluted with $30 \mathrm{ml}$ of $100 \mathrm{~g} / \mathrm{l}$ formic acid. The eluate was evaporated under reduced pressure. The dried residue was dissolved in $0.2 \mathrm{ml}$ of water, and centrifuged at $5000 \mathrm{~g}$ for $5 \mathrm{~min}$. Either $10 \mu \mathrm{l}$ or $20 \mu \mathrm{l}$ of the solution was directly injected onto the isotachophoretic analyser.

\section{Assay of glutathione}

Lens $(0.5 \mathrm{~g})$ and liver $(1.0 \mathrm{~g})$ were homogenized and centrifuged as described above. Each supernatant was analysed directly on an isotachophoretic analyser as reported previously (6).

\section{Instrumentation}

The capillary apparatus was a Shimadzu IP-3A isotachophoretic analyser, Shimadzu, Seisakusho, Kyoto, Japan.

The separations were carried out in a capillary tube, $200 \times 0.5 \mathrm{~mm}$ i. d., maintained at a constant temperature of $20^{\circ} \mathrm{C}$. The detector cell had an i.d. of $0.5 \mathrm{~mm}$ and length of $0.05 \mathrm{~mm}$. The migration current was $100 \mu \mathrm{A}$. The leading electrolyte consisted of 0.01 $\mathrm{mol} / \mathrm{l}$ hydrochloric acid and $\beta$-alanine $(\mathrm{pH} 2.8)$. The terminating electrolyte was $0.01 \mathrm{~mol} / \mathrm{l}$ caproic acid and chart speed was 10 or $20 \mathrm{~mm} / \mathrm{min}$.

\section{Analysis of amino acids}

Lenses from normal or naphthalene-treated rabbits were homogenized in four volumes of sulphosalicylic acid, and the homogenate centrifuged at $8000 \mathrm{~g}$. The supernatant was applied to a column containing $5 \mathrm{ml}$ of Diaion SA-100 (OH form), washed with water, and eluted with $2 \mathrm{~mol} / \mathrm{l}$ hydrochloric acid.

The eluate was dried under reduced pressure, and the residue dissolved in $0.02 \mathrm{~mol} / \mathrm{l}$ hydrochloric acid.

An aliquot of the solution was analysed using an amino acid analyser (Hitachi Model 835 liquid chromatograph).

\section{Cataract formation}

Male and female albino rabbits were divided into three groups, the normal group, the cataract developing group and the non-cataract developing group.

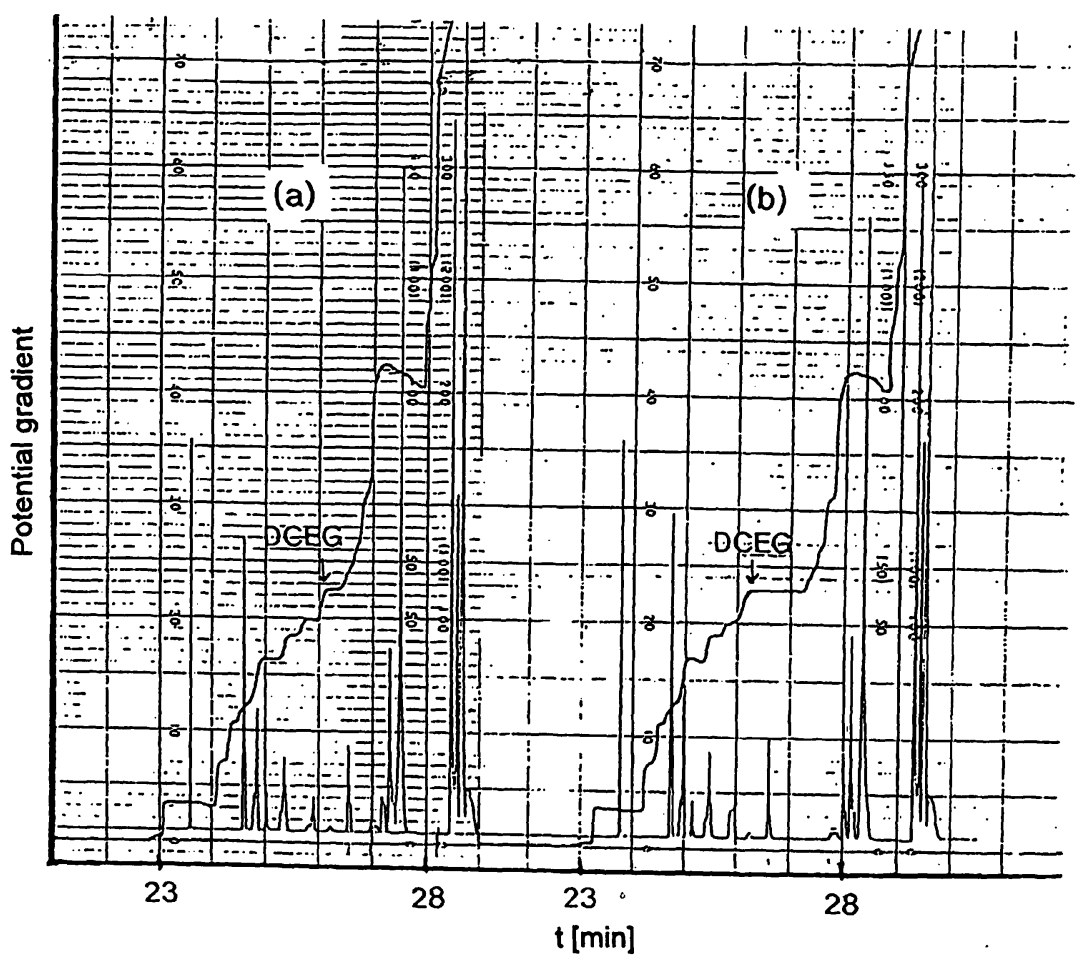

Fig. 2 Isotachophoretic runs of a sample of rabbit lens (a) and the same sample fortific with authentic S-(1,2-dicarboxyethyl)GSH (10 nmol) (b). 
Naphthalene $(2.5 \mathrm{~g} / \mathrm{kg}$ body weight) dissolved in $30 \mathrm{ml}$ of liquid paraffin was administered orally to rabbits of each group on the first day or for three consecutive days.

Naphthalene cataracts were classified into the two stages of spichen and true opacity. The stage of spichen developed 24 hours after the first administration. It was characterized by the appearance of axialed opacity "spichen" at the equatorial region of the lens.

The stage of true opacity developed a few weeks after the first administration.

The group that received only one administration did not develop to the stage of true opacity; this group is the non-cataract developing group. The group that developed to the stage of true opacity is the cataract developing group.

\section{Results and Discussion}

Figure $1 \mathrm{a}$ and $\mathrm{lb}$ show the isotachophoretic runs of authentic S-(1,2-dicarboxyethyl)glutathione, S-(1,2-dicarboxyethyl)-L-cysteine, GSH and GSSG.
Under the conditions described in figure 1, GSH and GSSG were easily separated as reported previously (6), but the separation of S-(1,2-dicarboxyethyl)glutathione and S-(1,2-dicarboxyethyl)- $L$-cysteine was unsatisfactory, since these two compounds ran together as a mixed zone. They were separated, however, by changing the $\mathrm{pH}$ of the leading electrolyte from 3.1 to 2.8 as shown in figure $1 b$.

The calibration curves for different concentrations of S(1,2-dicarboxyethyl)GSH and GSH showed linearity over the concentration ranges from 0 to $25 \mathrm{nmol}$. Figure $2 a$ and $2 b$ show the isotachophoretic runs corresponding to the sample of lens from normal rabbit and the same sample fortified with S-(1,2-dicarboxyethyl)GSH. The zone of S-(1,2-dicarboxyethyl)GSH in figure $2 \mathrm{a}$ was made to overlap by addition of authentic S-(1,2-dicarboxyethyl)GSH to the lens sample, resulting in an elon-

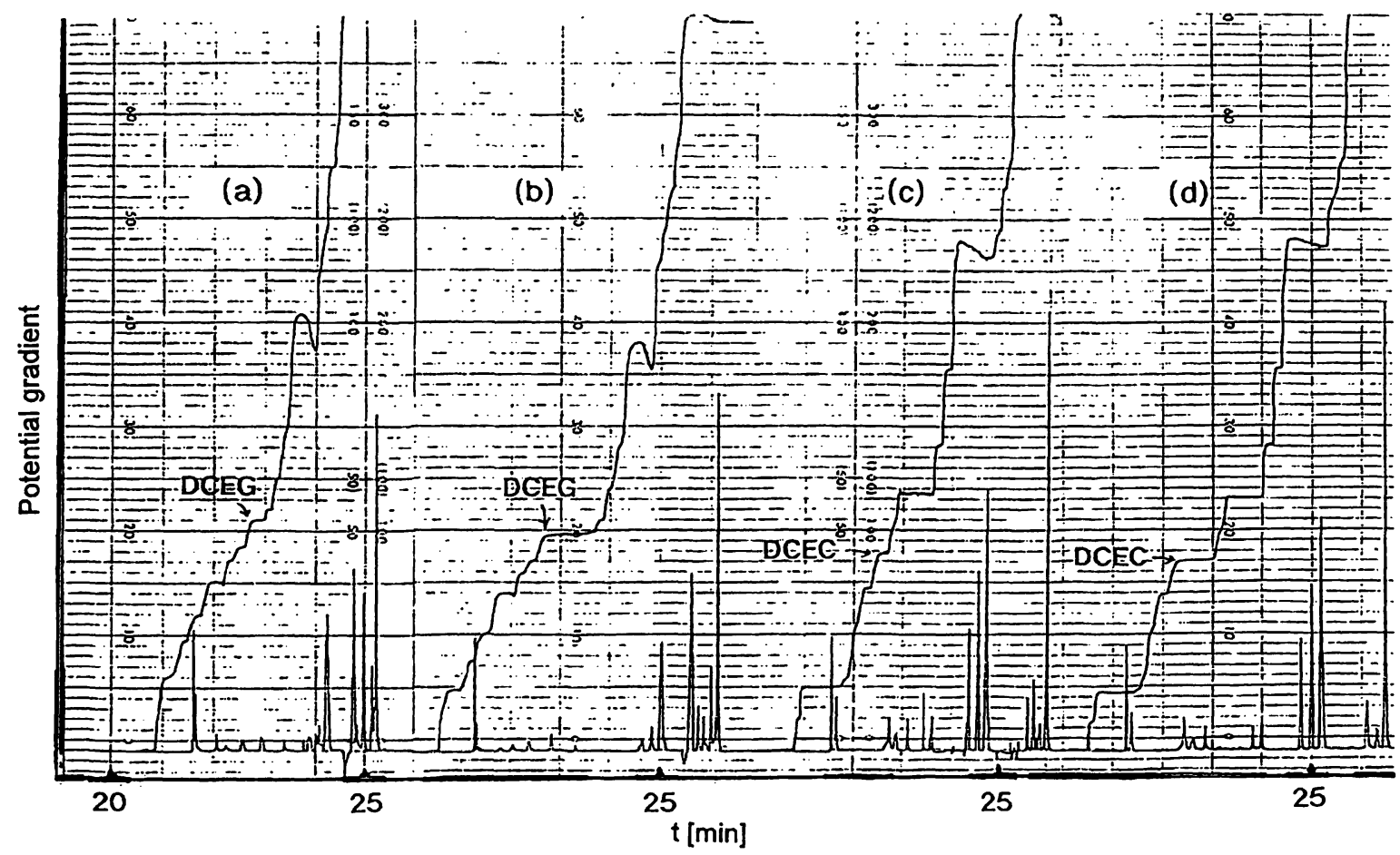

Fig. 3 Isotachophoretic runs of a sample of rabbit lens (a), a + S-(1,2-dicarboxyethyl)GSH (b), hydrolysate of a (c) and c + S-(1,2-dicarboxyethyl)-L-cysteine (d).

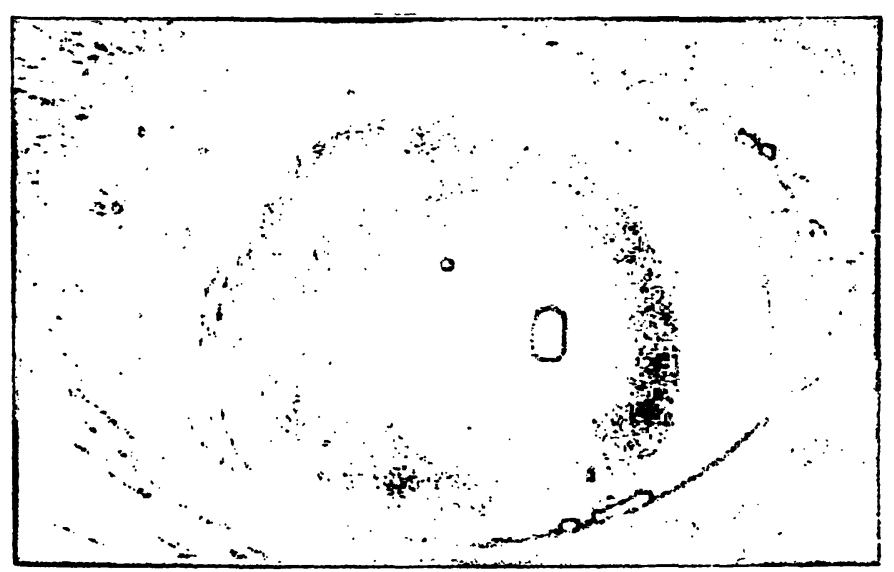

a

Fig. 4 Cataract at the spichen stage developed 24 hours after the first naphthalene administration (a) and cataract at the true

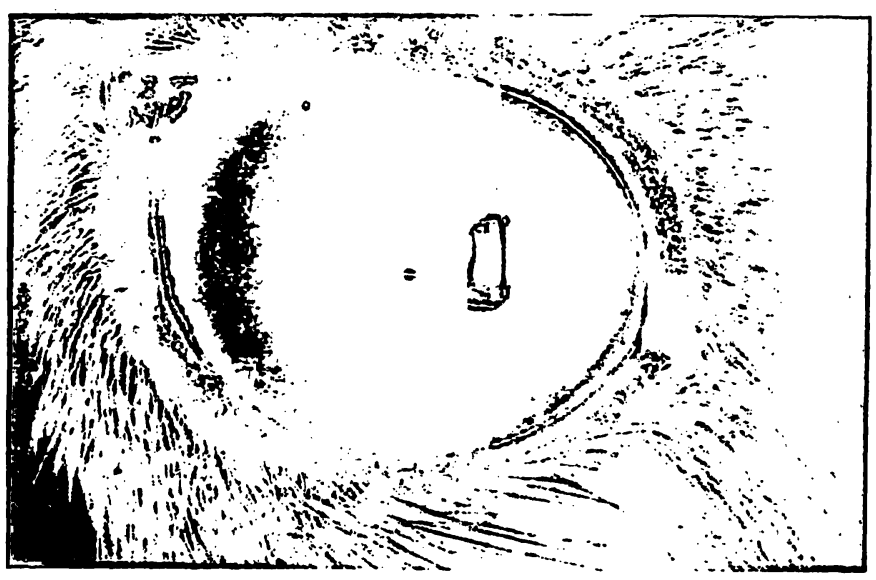

b

opacity stage developed a few weeks after naphthalene administration (b). 
gation of the zone of S-(1,2-dicarboxyethyl)GSH in the lens sample as shown in figure $2 b$.

The lens sample containing S-(1,2-dicarboxyethyl)GSH was hydrolysed in $6 \mathrm{~mol} / \mathrm{l}$ hydrochloric acid for 24 hours at $110^{\circ} \mathrm{C}$, the hydrolysate was evaporated under reduced pressure and the dried residue was injected onto the isotachophoretic analyser.

After hydrolysis, the zone of S-(1,2-dicarboxyethyl)GSH in the lens sample disappeared completely and was detected as a zone of S-(1,2-dicarboxyethyl)- $L$ cysteine (fig. 3c).

The zone of $S$-(1,2-dicarboxyethyl)- $L$-cysteine in figure $3 c$ was made to overlap by addition of authentic S-(1,2dicarboxyethyl)- $L$-cysteine to the hydrolysate of lens sample, resulting in an elongation of the zone of S-(1,2dicarboxyethyl)- $L$-cysteine in the hydrolysate as shown in figure $3 \mathrm{~d}$.

These findings indicate that the zone of S-(1,2-dicarboxyethyl)GSH in the lens sample in a single zone, and the amount of S-(1,2-dicarboxyethyl)GSH in the lens sample can be determined by measuring the zone length. Various amounts of S-(1,2-dicarboxyethyl)GSH were added to

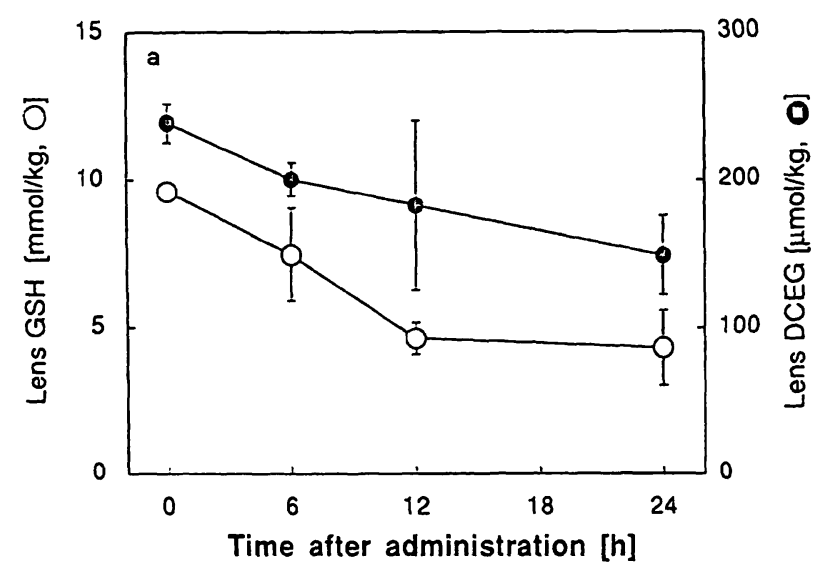

Fig. 5 Changes of GSH and S-(1,2-dicarboxyethyl)GSH (DCEG) in the lens during the 24 hours after naphthalene administration (a), and contents of GSH and S-(1,2-dicarboxyethyl)GSH in nor-



Fig. 6 Changes of GSH in the liver during the 24 hours after naphthalene administration (a), and contents of GSH in normal liver (control), in the liver in the cataract developing group (cata- rabbit liver homogenate and their recoveries were measured in a separate run by the procedure described above. The recovery of S-(1,2-dicarboxyethyl)GSH in rabbit liver after cation exchange resin treatment was 96.8 $\pm 11.3 \%(n=3)$. The contents of S-(1,2-dicarboxyethyl)GSH in normal rabbit lens and liver were 219.9 $\pm 29.1(\mathrm{n}=5)$ and $44.0 \pm 13.5(\mathrm{n}=8) \mathrm{nmol} / \mathrm{g}$, respectively.

The results of the determination of S-(1,2-dicarboxyethyl)GSH in normal rabbit lens and liver reported in this paper are in good agreement with an earlier report (5). The findings described above demonstrate that the method for measurement of S-(1,2-dicarboxyethyl)GSH using an isotachophoretic analyser was satisfactory. This isotachophoretic method offers several advantages over previously described techniques.

We studied the changes in the levels of S-(1,2-dicarboxyethyl)GSH and GSH in the rabbit lens and liver during the naphthalene cataract formation. Naphthalene at a dose of $2.5 \mathrm{~g} / \mathrm{kg}$ body weight was administered orally to rabbits.

Naphthalene cataracts were classified into the two stages of spichen and true opacity. Cataracts at the spichen

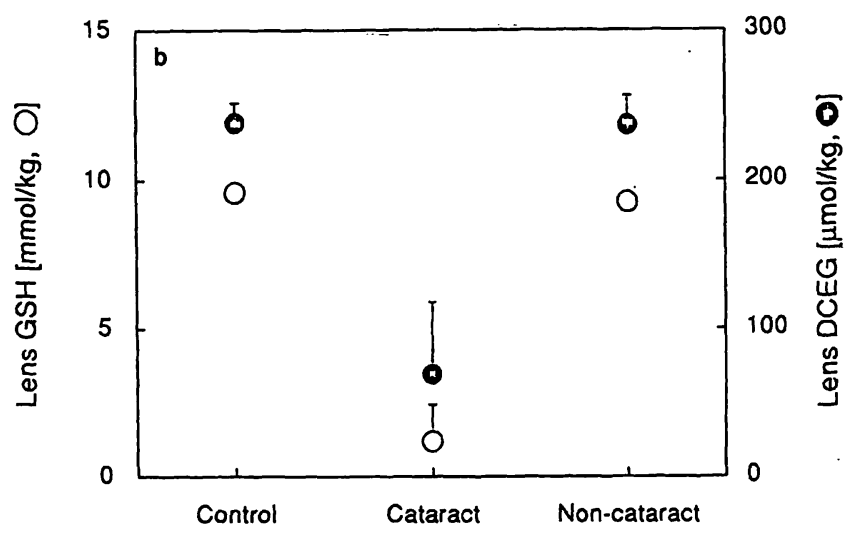

mal lens (control), in the lens in the cataract developing group (cataract) and in the lens in the non-cataract developing group (non-cataract) (b).

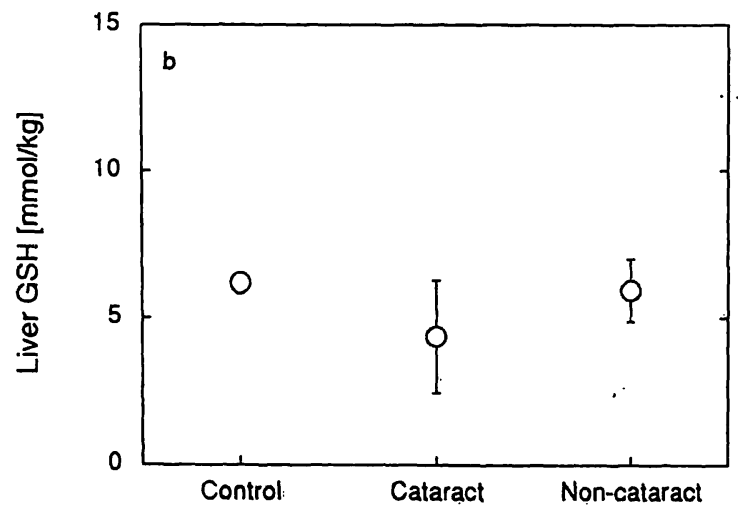

ract) and in the liver in the non-cataract developing group (noncataract) (b). 
Tab. 1 Contents of amino acids in lens of normal rabbits and rabbits after naphthalene feeding (n mol/g).



A: spichen developing lens at 24 hours after naphthalene administration.

B: cataract developing lens at a few weeks after naphthalene administration (three times).

stage developed 24 hours after the first administration. They were characterized by appearance of axialed opacity "spichen" at the equatorial region of lens (fig. 4a). Cataracts of the true opacity stage developed a few weeks after administration for three consecutive days (fig. 4b), but the rabbit which had received only once administration did not develop true opacity cataracts.

We examined the time course of the levels of S-(1,2dicarboxyethyl)GSH and GSH for 24 hours after naphthalene administration. Results are shown in figure $5 \mathrm{a}$.

The content of S-(1,2-dicarboxyethyl)GSH in the lens decreased until 24 hours after naphthalene administration in proportion to the content of GSH.

Raddy et al. (7) reported that the level of GSH in rat lens decreased rapidly during cataract formation, and S(1,2-dicarboxyethyl)GSH in rat lens also decreased rapidly during cataract formation by feeding galactose (5).

During the further development of true lens opacity after naphthalene administration, the S-(1,2-dicarboxyethyl)GSH content decreased further compared with that in the spichen stage, but the lens content of S-(1,2-dicarboxyethyl)GSH in the group that did not develop cataract after naphthalene administration returned to the normal level (fig. 5b).

The content of GSH in liver decreased markedly until 24 hours after naphthalene administration (fig. 6a).

The content of GSH in the liver in both the cataract developing group and the non-cataract developing group returned to the normal level (fig. 6b).
C: non-cataract developing lens at a few weeks after naphthalene administration (three times).

Table 1 shows the contents of amino acids in the lens of normal and naphthalene-treated rabbits.

The levels of the sum of reduced and oxidized glutathione, taurine and other free amino acids in the lens in the spichen stage decreased markedly compared with their levels in the lens of normal rabbits, while the levels of glutathione and taurine in the lens of the cataract developing rabbits a few weeks after naphthalene administration remained lower than those in the normal lens; but the levels of other amino acids returned to normal. On the other hand, glutathione, taurine and other amino acids in the lens in the non-cataract developing rabbits after naphthalene administration recovered to the same levels as those in the normal lens. It has been reported that the free amino acids are decreased in the lens of rats with galactose-generated cataract $(11,12)$.

In conclusion, these findings indicate that the changes in concentration of S-(1,2-dicarboxyethyl)GSH and GSH in the lens of naphthalene-treated rabbits are similar to those reported during cataract formation after galactose administration (5). The findings also indicate that the ratio of the synthesis and the degradation of GSH in rabbit lenses during cataract formation after naphthalene administration may be the same as that of S-(1,2-dicarboxyethyl)GSH.

The effect of GSH on prevention of cataract formation has been reported in previous papers $(11,12)$, but these findings suggest that taurine and $\mathrm{S}$-(1,2-dicarboxyethyl)GSH may also prevent cataract formation in the lens of rabbits. 


\section{References}

1. Calam DH, Waley SG. Acidic peptides of the lens. 8. S-( $\alpha \beta-$ dicarboxyethyl) glutathione. Biochem J 1963; 86:226-31.

2. Kuwaki T, Mizuhara S. S-(1,2-dicarboxyethyl) cysteine in urine and kidney. Biochim Biophys Acta 1966; 115:491-3.

3. Azumi T. Studies on nitrogen pool of animal tissues. I. Ox ocular tissues. II. Ox nervous tissues. Acta Med Okayama 1967; 21:315-20.

4. Azumi T. Studies on nitrogen pool of animal tissues. III. Ox liver and bile. IV. Ox kidney and lung. Acta Med Okayama 1967; 21:321-6.

5. Tsuboi S, Uda N, Ikeda M, Hirota K, Ohmori S. S-(1,2-dicarboxyethyl)glutathione and S-(1,2-dicarboxyethyl) $L$-cysteine in lens. J Clin Chem Clin Biochem 1984; 22:285-90.

6. Mikasa H, Ageta T, Mizobuchi N, Kodama H. Determination of glutathione and glutathione disulfide in rat tissues using isotachophoretic analyzer. Anal Biochem 1982; 126:52-7.

7. Arlinger L. Analytical isotachophoresis in capillary tubes. Analysis of hemoglobin, hemoglobin cyanide and isoelectric fractions of hemoglobin cyanide. Biochim Biophys Acta 1975; 393:396-403.
8. Kodama H, Uasa S. Isotachophoretic analysis of isovalerylglycerine in urine of a patient with isovaleric acidemia. J Chromatogr 1979; 163:300-3.

9. Miyazaki H, Katoh K. Isotachophoretic analysis of peptides. J Chromatogr 1976; 119:369-83.

10. Reddy VN, Schwass D, Chakrapani B', Lim CP. Biochemical changes associated with the development and reversal of galactose cataracts. Exp Eye Res 1976; 23:483-93.

11. Reddy DVN. Amino acid transport in the lens relation to sugar cataracts. Invest Ophthalmol 1965; 4:700-8.

12. Kinoshita JH, Barber GW, Merola LO, Tung B. Changes in the levels of free amino acids and myo-inositol in the galactose-exposed lens. Invest Ophthalmol 1969; 8:625-32.

Received June 13/August 25, 1995

Corresponding author: Prof. Hiroyuki Kodama, Department of Chemistry, Kohasu, Oko-cho, Nankoku-shi, Kochi 783, Japan 